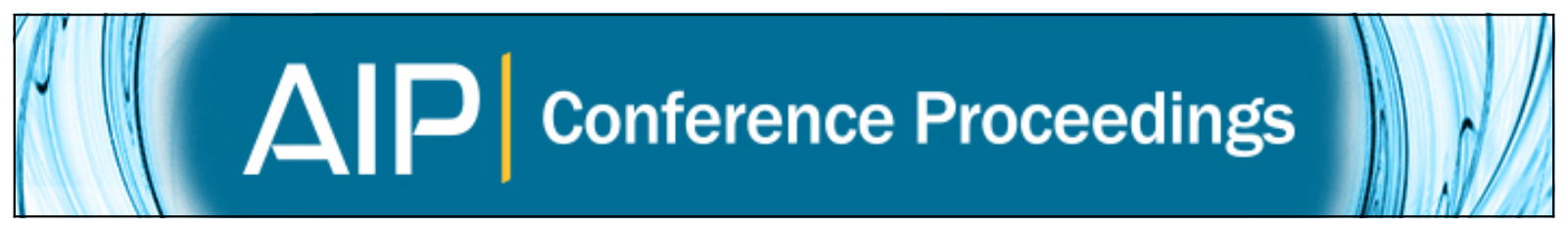

\title{
Extended Gauge Principle and Quantization of Gauge Theories
}

Gabriel Catren and Jorge A. Devoto

Citation: AIP Conference Proceedings 861, 300 (2006); doi: 10.1063/1.2399588

View online: http://dx.doi.org/10.1063/1.2399588

View Table of Contents: http://scitation.aip.org/content/aip/proceeding/aipcp/861?ver=pdfcov

Published by the AIP Publishing

\section{Articles you may be interested in}

BRST detour quantization: Generating gauge theories from constraints

J. Math. Phys. 51, 062302 (2010); 10.1063/1.3372732

The osp(1,2)-covariant Lagrangian quantization of reducible massive gauge theories J. Math. Phys. 40, 6189 (1999); 10.1063/1.533086

The osp $(1,2)$-covariant Lagrangian quantization of irreducible massive gauge theories J. Math. Phys. 40, 674 (1999); 10.1063/1.532681

Functional versus canonical quantization of a nonlocal massive vector-gauge theory

J. Math. Phys. 40, 585 (1999); 10.1063/1.532677

Quantization of minisuperspaces as ordinary gauge systems

J. Math. Phys. 39, 3131 (1998); 10.1063/1.532243 


\title{
Extended Gauge Principle and Quantization of Gauge Theories
}

\author{
Gabriel Catren* and Jorge A. Devoto ${ }^{\dagger}$ \\ *Instituto de Astronomía y Física del Espacio C.C. 67, Sucursal 28, 1428 Buenos Aires, Argentina \\ ${ }^{\dagger}$ Depto de Matemáticas, FCEN Universidad de Buenos Aires - Ciudad Universitaria 1428 Buenos Aires, \\ Argentina
}

Keywords: Gauge principle, BRST cohomology

PACS: $11.15 .-\mathrm{q}$ edited by J.-M. Alimi and A. Füzfa

(C) 2006 American Institute of Physics 978-0-7354-0359-8/06/\$23.00 


\section{INTRODUCTION}

The historical importance of General Relativity goes far beyond the fact that it provided a relativistic theory of gravitation. General Relativity was also the beginning of a general program of geometrization of the rest of the fundamental interactions. In the context of the standard model this program was performed by means of Yang-Mills theories. The guiding principle of these kinds of theories is the so-called gauge principle. The theories which satisfy this principle are called gauge theories. The main characteristic of gauge theories is the presence of constraints between the canonical variables. These theories emerge from theories with a global symmetry, which is revealed by the presence of conserved quantities through the first Noether's theorem. The constraints appear as a consequence of the localization of these global symmetries (second Noether's theorem). The fundamental fact is that by demanding the invariance of the theory under these kinds of local coordinate transformations, the relevant fields carrying the fundamental interactions appear naturally as nontrivial geometric structures (namely connections) of the corresponding fiber bundles over space-time. These new geometric degrees of freedom are called gauge fields.

In this contribution, we present a review of the formalism developed in Ref.[5] concerning the study of the geometric structure and quantization of Yang-Mills theories by using what we have called an extended gauge principle. In general, the localization of a theory with a global symmetry not only requires the definition of gauge fields, but also demands other new geometric constructions. Firstly, it is necessary to fix the gauge by means of a section of the corresponding principal bundle. Roughly speaking, fixing the gauge amounts to choosing in a continuous way a coordinate system at the fibers for each point of space-time. Secondly, it is necessary to know how to extract from the resulting theory gauge invariant information (observables). This can be done by means of the so-called BRST formalism (see Ref.[10]). This formalism requires an extension of the set of canonical variables by adding the so-called ghost fields.

In Ref.[5] it was shown that these fundamental geometric components of Yang-Mills theories - the gauge fields, the gauge fixing and the ghost fields - can be considered as different aspects of a single geometric object: an extended connection in a properly chosen principal bundle. This unification is not only a conceptual improvement, but also provides a means of solving the so-called Gribov ambiguity in the quantization of Yang-Mills theories (see Refs. $[8,11])$. In fact, in order to construct the extended connection it is necessary to generalize the notion of gauge fixing by using a gauge fixing connection instead of the usual local sections. A fundamental difference is that the gauge fixing connection is globally well defined, even when the topology of the fiber bundle is not trivial (Gribov ambiguity). It was also shown in Ref.[5] that it is possible to derive the corresponding BRST transformations from the equations for the curvature of the extended connection, without imposing the usual horizontality conditions used for passing from topological Yang-Mills theories to ordinary Yang-Mills theories (see for example Ref.[3]). Finally, it is shown that it is possible to follow the Faddeev-Popov method in order to find the gauge fixed action corresponding to the generalized gauge fixing.

\section{YANG-MILLS THEORIES}

As we will see later, the kind of gauge fixing that we will use can be naturally implemented in the hamiltonian, or canonical, formulation of Yang-Mills theories. We will then suppose that the space-time manifold $\mathscr{M}$ can be foliated by space-like hypersurfaces. In other words, we shall assume that the space-time $\mathscr{M}$ is diffeomorphic to a product space $\mathbb{R} \times M$ with $M$ a 3-dimensional Riemannian manifold that we will assume compact for simplicity.

In this framework, Yang-Mills theories are defined in a $G$-principal fiber bundle $P \rightarrow M$, where $G$ is a compact Lie group with Lie algebra $\mathfrak{g}$. The usual gauge principle states that in order to render the theory invariant under local gauge transformations generated by the gauge group $\mathscr{G}$ of vertical automorphisms of $P{ }^{1}$, it is necessary to introduce connections $A$, which can be considered as $\mathfrak{g}$-valued 1 -forms on $P$, i.e. as an element of $\Omega^{1}(P) \otimes \mathfrak{g}$. In a local trivialization defined by a local section $s: U \rightarrow P$, where $U$ is an open subset of $M$, the connection $A$ can be identified with the usual gauge fields $A_{\mu}^{a} \in \Omega^{1}(U) \otimes \mathfrak{g}$.

Let $\mathscr{A}$ be the configuration space of all connections in $P \rightarrow M$. This space is an affine space. The action of the gauge group $\mathscr{G}$ on $P$ induces an action on $\mathscr{A}$. On each connection $A$ the action is given by the usual formula $A^{\prime}=g^{-1} A g+g^{-1} d g$. This action defines a structure of a $\mathscr{G}$-principal fiber bundle $\mathscr{A} \stackrel{\pi}{\rightarrow} \mathscr{A} / \mathscr{G}$, where $\mathscr{A} / \mathscr{G}$ is the

\footnotetext{
1 The gauge group $\mathscr{G}$ can be identified with the sections of the adjoint bundle ad $(P)=P \times{ }_{G} G$ and its Lie algebra $\mathscr{L}$ ie $(\mathscr{G})$ with the sections of $P \times_{G} \mathfrak{g}$. When the fiber bundle $P \rightarrow M$ is trivial, the elements of this group can be considered as maps $g(x): M \rightarrow G$. In other words, an element of $\mathscr{G}$ defines at each $x \in M$ a coordinate transformation given by $g(x) \in G$. This is the usual description of $\mathscr{G}$.
} 
orbit space of connections modulo gauge transformations ${ }^{2}$. Each orbit $\pi^{-1}([A])$ (with $[A] \in \mathscr{A} / \mathscr{G}$ ) of this fiber bundle is a set of gauge equivalent connections.

Since the geometric structure of the orbit space $\mathscr{A} / \mathscr{G}$ is generally very complicated, it is much more convenient to work on $\mathscr{A}$. The usual means of doing so is by fixing the gauge by defining a gauge fixing section $\sigma$ :

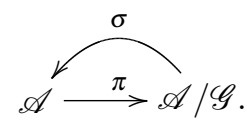

For each equivalence class $[A] \in \mathscr{A} / \mathscr{G}$, the gauge fixing section $\sigma$ selects a unique representative $A \in \mathscr{A}$ in the corresponding orbit $\pi^{-1}([A])$. The problem is that in Yang-Mills theories it is generally impossible to define a global gauge fixing section $\sigma$, given that the topology of the fiber bundle $\mathscr{A} \rightarrow \mathscr{A} / \mathscr{G}$ is not necessarily trivial. This is the so-called Gribov ambiguity (see Refs.[8, 11]).

Once the theory is gauge fixed, the original gauge invariance is no longer manifest. Nevertheless, it was discovered that the gauge fixed theory still has a rigid symmetry, namely the BRST symmetry [10]. This symmetry is implemented by a nilpotent operator of order two, which defines a complex with its corresponding BRST cohomology. The generators of this complex are the so-called ghost fields. As it was shown in Ref.[4] the ghost fields can be identified with the Maurer-Cartan forms $\theta_{M C}$ of the gauge group $\mathscr{G}$, which are the canonical 1-forms on $\mathscr{G}$ with values in its Lie algebra $\mathscr{L} i e(\mathscr{G})$. The fundamental point is that the BRST differential $\delta_{B R S T}$ is constructed in such a way that its zero degree cohomology coincides with the set of observables of the theory

$$
H^{0}\left(\delta_{B R S T}\right)=\{\text { Observables }\}
$$

i.e. with the set of gauge invariant functions on the reduced phase space (constraint surface modulo gauge transformations).

We can then say that Yang-Mills theories contain three fundamental geometric objects: gauge fields, gauge fixing section, and ghost fields.

\section{THE EXTENDED CONNECTION}

The question that we want to answer is whether there exists a single geometric entity which unifies these three components of Yang-Mills theories. The answer proposed in Ref.[5] is that it is in fact possible to define such an entity, which is found to be an extended connection $\mathbb{A}$ in a properly chosen principal fiber bundle. The main references for certain aspects of the following geometric construction are the seminal papers of M.F. Atiyah and I.M. Singer [1] and L. Baulieu and I.M. Singer [2].

The first step of this unification process is the definition of a connection in the fiber bundle $\mathscr{A} \rightarrow \mathscr{A} / \mathscr{G}$. Such a connection can be defined by means of a $\mathscr{L} i e(\mathscr{G})$-valued 1-form $\eta$ on $\mathscr{A}$ which must satisfy a certain equivariance condition under the action of $\mathscr{G}$. At each point $A \in \mathscr{A}$, the connection 1-form $\eta$ defines a horizontal subspace $\mathscr{H}_{A}^{\eta}$ of the tangent space $T \mathscr{A}_{A}$ through its kernel: $\mathscr{H}_{A}^{\eta}=\operatorname{Ker} \eta_{A}=\left\{X \in T \mathscr{A}_{A} \mid \eta_{A}(X)=0\right\}$. In other words, a connection can be also considered as an equivariant horizontal distribution. The fundamental point is that the connection $\eta$ in the fiber bundle $\mathscr{A} \rightarrow \mathscr{A} / \mathscr{G}$ will encode both the ghost fields and a generalized notion of gauge fixing.

The main observation is that connections can be considered as infinitesimal versions of sections. Given any global gauge fixing section $\sigma$, one can always define an induced flat connection $\eta_{\sigma}$ such that the section $\sigma$ is horizontal for $\eta_{\sigma}$. This means that the tangent spaces to the section coincide with the horizontal subspaces defined by the connection. On the contrary, a connection can not generally be integrated, even locally, to a section. A local obstruction is the curvature and a global one is the monodromy. The fundamental difference between a connection and a section is that, even when the topology of the fiber bundle $\mathscr{A} \rightarrow \mathscr{A} / \mathscr{G}$ is not trivial (Gribov ambiguity), it is always possible to define a global gauge fixing connection $\eta$.

The main use of the connection $\eta$ will be to define a section not on the level of $\mathscr{A} \rightarrow \mathscr{A} / \mathscr{G}$ but on the level of paths. The section will be defined by the parallel transport along paths $\gamma$ in $\mathscr{A} / \mathscr{G}$ with any given fixed initial condition $A_{0} \in \mathscr{A}$. We shall see that this section is what is necessary in order to quantize the theory using path integrals.

\footnotetext{
2 Technically speaking we shall work with Sobolev framed connections (see Ref.[6]). In this way one can assure that the action of the gauge group $\mathscr{G}$ on $\mathscr{A}$ is free and one also gets local triviality. We will then consider that the space $\mathscr{A}$ is the configuration space of framed connections
} 
It can be shown that the gauge fixing connection $\eta$ encodes also the ghost fields. A local section $\sigma: U \subset \mathscr{A} / \mathscr{G} \rightarrow \mathscr{A}$ induces a trivialization of the fiber bundle $\mathscr{A} \rightarrow \mathscr{A} / \mathscr{G}$. This means that the restriction to $U$ of the fiber bundle $\mathscr{A} \rightarrow \mathscr{A} / \mathscr{G}$ can be identified with a trivial product $U \times \mathscr{G}$. This identification is given by a map $\varphi_{\sigma}: U \times \mathscr{G} \rightarrow \pi^{-1}(U)$ defined as $\varphi_{\sigma}([A], g)=\sigma([A]) g$. It is important to remark that this local identification is non canonical because it depends on the arbitrary local section $\sigma$. Given this local map $\varphi_{\sigma}$, one can take the pullback of the connection $\eta$ (which is defined on $\pi^{-1}(U)$ ), i.e. we can look at it as a connection on the trivial product $U \times \mathscr{G}$. If we do so, we find that the connection $\varphi_{\sigma}^{*} \eta$ defined by the pullback can be decomposed in a 1 -form on the base space $U$ and a canonical vertical component which coincides with the Maurer-Cartan form $\theta_{M C}$ of the gauge group $\mathscr{G}$. If $w$ is a tangent vector to $U$ at $[A]$ and $v$ a tangent vector to $\mathscr{G}$ at $g$, the action of the connection 1 -form $\varphi_{\sigma}^{*} \eta$ on $w+v$ is given by

$$
\varphi_{\sigma}^{*} \eta(w+v)=g^{-1} \eta(w) g+\theta_{M C}(v)
$$

where $\eta=\sigma^{*} \eta \in \Omega^{1}(U) \otimes \mathscr{L} i e(\mathscr{G})$ (local form of the connection $\eta$ ). Given that the ghost field is the Maurer-Cartan form $\theta_{M C}$ of the gauge group $\mathscr{G}$ [4], this decomposition means that in a local trivialization we can identify the ghost field with the canonical vertical component of the connection $\eta$. In this sense, we could say that the ghost field and the gauge fixing of the theory are different aspects of the same geometric entity, namely the connection $\eta$ in the $\mathscr{G}$-principal fiber bundle $\mathscr{A} \rightarrow \mathscr{A} / \mathscr{G}$.

Nevertheless, this way of understanding the relation between the connection $\eta$ and the ghost field, even if it is clarifying, it is not fully satisfactory given that it depends on the definition of a family of local sections $\sigma$ (which is equivalent to fix the gauge in the usual way). Therefore, it would be much better if one could understand the relation between the connection $\eta$ and the ghost field without using such a family of local sections. This can be done if we take into account that a connection in a fiber bundle can be defined by means of a universal construction. The general philosophy is that a particular structure on an object can be defined by giving a particular morphism from (or to) a universal object to (or from) the object in question. In our case, this means that instead of defining a particular connection $\eta$ in the fiber bundle $\mathscr{A} \rightarrow \mathscr{A} / \mathscr{G}$ one can give a morphism from a universal algebraic object to the differential algebra of $\mathscr{L}$ ie $(\mathscr{G})$-valued forms in $\mathscr{A}$. The universal object in question is Weil algebra and the corresponding morphisms are Chern-Weil homomorphisms (see Refs.[7, 9]). Weil algebra is the abstract model which reproduces in an universal way the algebra of a connection and its curvature. The important fact is that the object which plays the role of the universal connection in Weil algebra is the Maurer-Cartan form of the gauge group $\mathscr{G}$ (i.e. the ghost field). In this way we can say that a particular Chern-Weil "immersion" $\omega_{\natural}$ of the ghost field $\theta_{M C}$ (universal connection) in the differential algebra $\Omega^{*}(\mathscr{A}) \otimes \mathscr{L}$ ie $(\mathscr{G})$ defines a particular gauge fixing connection $\eta=\omega_{\sharp}\left(\theta_{M C}\right)$. Different "incarnations" of the universal ghost $\theta_{M C}$ define in this way different gauge fixings of the theory.

We have then the $G$-principal fiber bundle $P \rightarrow M$ with the connection $A$ (i.e. with the gauge fields $A_{\mu}^{a}$ defined in a local trivialization) and the $\mathscr{G}$-principal fiber bundle $\mathscr{A} \rightarrow \mathscr{A} / \mathscr{G}$ with the connection $\eta$ (i.e. with the definition of the gauge fixing and the ghost field). In order to give the last step in the unification process, it is necessary to define a unified principal fiber bundle. We will do so by multiplying each point of the fiber bundle $P \rightarrow M$ by $\mathscr{A}$. It results the $G$-principal fiber bundle $\mathscr{A} \times P \rightarrow \mathscr{A} \times M^{3}$. This fiber bundle can be considered as a family (parameterized by the space of all connections $\mathscr{A}$ ) of $G$-principal fiber bundles $P \rightarrow M$ with a universal family $\mathbf{A}^{U}$ of connections $A$. This means that at each point $A$ in the space of all connections $\mathscr{A}$ there is a copy of the $G$-principal fiber bundle $P \rightarrow M$ with connection $A$. The universal family $\mathbf{A}^{U}$ can be considered as a universal gauge field which contains the information about all the particular fields.

In this way, we have a unified $G$-principal fiber bundle $\mathscr{A} \times P \rightarrow \mathscr{A} \times M$. We now wish to define a unified geometric structure on this principal fiber bundle. It can be shown that it is possible to define an extended connection form $\mathbb{A} \in \Omega^{1}(\mathscr{A} \times P) \otimes \mathfrak{g}$ on this fiber bundle which is the sum of the universal family $\mathbf{A}^{U}$ of connections $A$ in the fiber bundle $P \rightarrow M$ plus the connection form $\eta$ in the fiber bundle $\mathscr{A} \rightarrow \mathscr{A} / \mathscr{G}$. The action of the extended connection $\mathbb{A}$ on tangent vectors $v_{1}+v_{2} \in T_{A} \mathscr{A} \oplus T_{p} P$ is defined as

$$
\mathbb{A}\left(v_{1}+v_{2}\right)=\mathbf{A}_{(A, p)}^{U}\left(v_{2}\right)+\eta\left(v_{1}\right)(p) \in \mathfrak{g}
$$

\footnotetext{
${ }^{3}$ More formally, one can take the product $\mathscr{A} \times M$ and the pullback of the fiber bundle $P \rightarrow M$ by the projection $q: \mathscr{A} \times M \rightarrow M$. The total space of the resulting fiber bundle is $q^{*}(P)=\mathscr{A} \times P$.
} 
It can be shown that the horizontal distribution $\mathscr{H}_{\mathbb{A}}$ defined by the extended connection form $\mathbb{A}$ has three components:

- the horizontal subspaces $\mathscr{H}_{\eta}$ defined by the connection $\eta$ in the fiber bundle $\mathscr{A} \rightarrow \mathscr{A} / \mathscr{G}$,

- the horizontal subspaces $\mathscr{H}_{A}$ defined by the universal family $\mathbf{A}^{U}$ of connections in each copy of the fiber bundle $P \rightarrow M$ and

- the tangent space $T \mathscr{G}$ of the orbit of the action of the gauge group $\mathscr{G}$ on $\mathscr{A} \times P$.

The horizontal distribution $\mathscr{H}_{\mathbb{A}}$ can then be decomposed as

$$
\mathscr{H}_{\mathbb{A}}(A, p)=\mathscr{H}_{\eta}(A) \oplus \mathscr{H}_{A}(p) \oplus T \mathscr{G}(A, p) .
$$

The presence of the last term $T \mathscr{G}(A, p)$ shows that the gauge group $\mathscr{G}$, even if it comes from the factor $\mathscr{A}$, has to act on both $\mathscr{A}$ and $P$ in order to define a horizontal subspace. This means that this geometric construction is not just a trivial addition of connections in a product space.

\section{BRST SYMMETRY}

From the extended connection $\mathbb{A}$, we can calculate the associated extended curvature $\mathbb{F}=\Delta_{\mathbb{A}} \mathbb{A}$. Given that the total space $\mathscr{A} \times P$ is a product space, there is a natural bigraduation of the differential forms $((p, q)$-forms $\in$ $\left.\Omega^{p}(\mathscr{A}) \otimes \Omega^{q}(P)\right)$ and the total differential $\Delta$ can be decomposed in a differential $\delta$ in $\mathscr{A}$ and a differential $d$ in $P$. The extended curvature $\mathbb{F}$ has then three components

$$
\mathbb{F}=\mathbb{F}^{(2,0)}+\mathbb{F}^{(1,1)}+\mathbb{F}^{(0,2)}
$$

given by the expressions

$$
\begin{aligned}
\mathbb{F}^{(2,0)} & =\delta \eta+\frac{1}{2}[\eta, \eta] \equiv \phi \\
\mathbb{F}^{(1,1)} & =\delta \mathbf{A}^{U}+d \eta+\left[\mathbf{A}^{U}, \eta\right] \equiv \psi \\
\mathbb{F}^{(0,2)} & =d \mathbf{A}^{U}+\frac{1}{2}\left[\mathbf{A}^{U}, \mathbf{A}^{U}\right] \equiv \mathbf{F}^{U}
\end{aligned}
$$

The (0,2)-forms $\mathbf{F}^{U}$ are the universal family of curvature forms corresponding to the universal family of connections $\mathbf{A}^{U}$. The (2,0)-form $\phi$ is the curvature of the connection $\eta$ and the (1,1)-form $\psi$ is a mixed term which involves both the gauge fields $A$ and the gauge fixing connection $\eta$. This last term shows again that this geometric construction mixes in a non trivial way the geometric structures coming from the fiber bundles $P \rightarrow M$ and $\mathscr{A} \rightarrow \mathscr{A} / \mathscr{G}$.

Without imposing the usual horizontality conditions $\left(\phi=\psi=0\right.$ or equivalently $\left.\mathbb{F}=\mathbf{F}^{U}\right)$, it is demonstrated in Ref.[5] that the equations for $\psi$ and $\phi$ can be separated in horizontal and vertical components as

$$
\delta_{V} \mathbf{A}^{U}=-d_{\mathbf{A}^{U}} \eta \quad \delta_{H} \mathbf{A}^{U}=\psi
$$

and

$$
\delta_{V} \eta=-\frac{1}{2}[\eta, \eta] \quad \delta_{H} \eta=\phi
$$

If we take into account the relation between the connection $\eta$ and the ghost field, and if we identify the vertical differential $\delta_{V}$ with the BRST differential, the equations corresponding to the vertical components reproduce the BRST transformations of the gauge and ghost fields.

\section{PATH INTEGRAL QUANTIZATION OF YANG MILLS THEORIES}

We shall now outline how this formalism can be implemented at the level of the path integral. In order to introduce the generalized gauge fixing, we will consider the path integral as an integral over the space of paths

$$
\mathscr{P}\left(A_{0}, \pi^{-1}\left[A_{1}\right]\right)=\left\{\gamma:[0,1] \rightarrow \mathscr{A} \mid \gamma(0)=A_{0}, \pi(\gamma(1))=\left[A_{1}\right]\right\}
$$


between a fixed initial condition $A_{0} \in \mathscr{A}$ and any element belonging to a final fiber $\pi^{-1}\left[A_{1}\right]$. In other words, the boundary conditions are such that the final condition is defined only up to a gauge transformation. The transition amplitude that one wants to calculate takes then the form

$$
\left\langle A_{0} \mid \pi^{-1}\left[A_{1}\right]\right\rangle=\int_{T^{*} \mathscr{P}\left(A_{0}, \pi^{-1}\left[A_{1}\right]\right)} \exp \{i S\} \mathscr{D} \mathscr{A} \mathscr{D} \pi,
$$

where $S$ is the canonical Yang-Mills action

$$
S=\int d t \int d^{3} x\left(\dot{A}_{k}^{a} \pi_{a}^{k}-\mathscr{H}_{0}\left(\pi_{a}^{k}, B_{a}^{k}\right)-A_{0}^{a} \phi_{a}\right)
$$

As it is clear from the form of this action, the pairs $\left(A_{k}^{a}, \pi_{a}^{k}\right)$ are the canonical variables of the theory while $A_{0}^{a}$ is not a dynamical variable, but the Lagrange multiplier for the generalized Gauss constraint $\phi_{a} \approx 0^{4}$.

The action of the gauge group $\mathscr{G}$ on each connection $A$ - except for the fixed initial condition $A_{0}-$ defines an action on each path $\gamma$ in $\mathscr{P}\left(A_{0}, \pi^{-1}\left[A_{1}\right]\right)$ generated by the path group

$$
\mathscr{P} \mathscr{G}=\left\{p:[0,1] \rightarrow \mathscr{G} \mid p(0)=\mathrm{id}_{\mathscr{G}}\right\}
$$

of continues parameterizations of elements of the gauge group $\mathscr{G}$. The action of the path group $\mathscr{P} \mathscr{G}$ on $\mathscr{P}\left(A_{0}, \pi^{-1}\left[A_{1}\right]\right)$ defines a projection $\tilde{\pi}$

$$
\mathscr{P}\left(A_{0}, \pi^{-1}\left[A_{1}\right]\right) \stackrel{\tilde{\pi}}{\rightarrow} \mathscr{P}\left(\left[A_{0}\right],\left[A_{1}\right]\right)
$$

where

$$
\mathscr{P}\left(\left[A_{0}\right],\left[A_{1}\right]\right)=\left\{[\gamma]:[0,1] \rightarrow \mathscr{A} / \mathscr{G} \mid \gamma(i)=\left[A_{i}\right], i=0,1\right\}
$$

is the space of paths $[\gamma]$ in the orbit space $\mathscr{A} / \mathscr{G}$. This projection $\tilde{\pi}$ sends each path $\gamma$ going from $A_{0} \in \mathscr{A}$ to an element of the final fiber $\pi^{-1}\left[A_{1}\right]$ to the projected path $[\gamma]$ in the orbit space $\mathscr{A} / \mathscr{G}$.

In the general case (with curvature or monodromy), the connection $\eta$ does not induce a section of $\mathscr{A} \rightarrow \mathscr{A} / \mathscr{G}$, given that the parallel transports defined by $\eta$ are path depending. Nevertheless, parallel transports defined by $\eta$ along paths in $\mathscr{A} / \mathscr{G}$ of the initial condition $A_{0} \in \mathscr{A}$ defines always an induced section $\sigma_{\eta}$ of the projection $\tilde{\pi}$ in the space of paths:

$$
\mathscr{\mathscr { P }}\left(A_{0}, \pi^{-1}\left[A_{1}\right]\right) \stackrel{\sigma_{\eta}}{\stackrel{\tilde{\pi}}{\longrightarrow} \mathscr{P}}\left(\left[A_{0}\right],\left[A_{1}\right]\right) .
$$

In other words, the section $\sigma_{\eta}$ sends each path $[\gamma] \in \mathscr{P}\left(\left[A_{0}\right],\left[A_{1}\right]\right)$ to its $\eta$-horizontal lift $\gamma=\sigma_{\eta}([\gamma]) \in$ $\mathscr{P}\left(A_{0}, \pi^{-1}\left[A_{1}\right]\right)$ starting at $A_{0}$. The local condition for belonging to the section $\sigma_{\eta}$ is that the tangent vectors to $\gamma$ have to belong at each point $A \in \mathscr{A}$ to the horizontal subspace $\mathscr{H}_{\eta}(A)$ defined by the connection form $\eta$. Recalling that $\mathscr{H}_{\eta}(A)=\operatorname{Ker} \eta(A)$, this condition leads to the gauge fixing equation

$$
\eta(\dot{\gamma}(t))=0, \quad \forall t
$$

Taking into account that strictly speaking the path integral is an integral on the space of paths (and not on the space of fields), the section $\sigma_{\eta}$ induced by the gauge fixing connection $\eta$ suffices for getting rid of the infinite volume of the gauge group $\mathscr{G}$ in the path integral.

In Ref.[5] it was shown that in order to introduce this generalized gauge fixing in the path integral, it is possible to follow the Faddeev-Popov method. It is then necessary to introduce a Dirac's delta of the gauge fixing condition $\eta(\dot{\gamma})=$ 0 and the determinant of the operator which measures the variation of this gauge condition under an infinitesimal gauge transformation. The Dirac's delta of the gauge fixing condition admits an integral representation of the form

$$
\delta(\eta(\dot{\gamma}))=\int \mathscr{D} \lambda e^{i F}, \quad \text { with } F=\int_{\gamma} \int_{M} \lambda_{a} \eta(\dot{\gamma}(t))^{a} d^{3} x d t
$$

\footnotetext{
${ }^{4}$ In the covariant formalism, $A_{0}^{a}$ is the temporal component of the connection $\mathscr{A}=A(t)+A_{0}(t) d t$ in the corresponding fiber bundle $\mathscr{P} \rightarrow \mathscr{M}$ over the space-time.
} 
where $\lambda$ is a time-evolving section of $\operatorname{ad}(P)=P \times_{G} \mathfrak{g}$ and the measure $\mathscr{D} \lambda$ is

$$
\mathscr{D} \lambda=\lim _{N, M} \prod_{k=1}^{N} \prod_{j=1}^{M} d \lambda_{k}\left(x_{j}\right)
$$

In Ref.[5] it is demonstrated that the variation of the gauge condition under an infinitesimal gauge transformation defines for each path $\gamma(t)$ an endomorphism $M_{\gamma}(t)$ in $\mathscr{L}$ ie $(\mathscr{G})$ given by

$$
M_{\gamma}(t)(x)_{a}^{c}=-\eta_{\gamma(t)}(\dot{\gamma}(t))^{b} f_{a b}^{c}+\delta_{a}^{c} \partial_{0}
$$

where $f_{a b}^{c}$ are the structure constants of the Lie algebra $\mathfrak{g}$ of $G$. By introducing the usual Grassmann's variables $(c, \bar{c})$, the corresponding Faddeev-Popov determinant can be expressed as

$$
\Delta[\gamma]=\int \mathscr{D} \bar{c} \mathscr{D} c e^{S}, \quad \text { where } S=\int \bar{c}^{a} M_{\gamma}(t)_{a}^{c} c_{c} d^{3} x d t
$$

where

$$
\mathscr{D} c=\lim _{N, M} \prod_{k=1}^{N} \prod_{j=1}^{M} d c_{t_{k}}\left(x_{j}\right)
$$

and the same for $\mathscr{D} \bar{c}$.

The gauge fixed action $S_{g f}$ takes then the form

$$
S_{g f}=\int d^{4} x\left(\dot{A}_{k}^{a} \pi_{a}^{k}-\mathscr{H}_{0}-A_{0}^{a} \phi_{a}+\lambda_{a} \eta(\dot{\gamma})^{a}+i \bar{c}^{a} \eta(\dot{\gamma})^{b} f_{a b}^{c} c_{c}-i \bar{c}^{a} \dot{c}_{a}\right)
$$

In this way the existence of a generalized notion of gauge fixing shows that the Faddeev-Popov method is still valid even in the presence of a Gribov ambiguity.

\section{CONCLUSIONS}

An extended connection $\mathbb{A}=\mathbf{A}^{U}+\eta$ was defined in the $G$-principal fiber bundle $\mathscr{A} \times P \rightarrow \mathscr{A} \times M$. To do so it it was necessary to generalize the notion of gauge fixing from a gauge fixing section $\sigma$ to a gauge fixing connection $\eta$. A fundamental difference is that the gauge fixing connection $\eta$ is globally well defined, even when the topology of the $\mathscr{G}$-principal fiber bundle $\mathscr{A} \rightarrow \mathscr{A} / \mathscr{G}$ is not trivial (Gribov ambiguity).

In this way, the single geometric object $\mathbb{A}$ unifies the three fundamental components of Yang-Mills theories, namely the gauge fields (connections $A$ ), the gauge fixing (connection $\eta$ ) and the ghost field (universal connection or canonical vertical component of $\eta$ in a local trivialization):

$$
\text { Extended connection } \mathbb{A}=\mathbf{A}^{U}+\eta\left\{\begin{array}{l}
\text { A Gauge fields } \\
\eta\left\{\begin{array}{l}
\text { Ghosts } \\
\text { Generalized gauge fixing. }
\end{array}\right.
\end{array}\right.
$$

From the curvature $\mathbb{F}=\Delta_{\mathbb{A}} \mathbb{A}$ of the extended connection $\mathbb{A}$ we derived the BRST transformations of the gauge and ghost fields without imposing the usual horizontality conditions $\phi=\psi=0$. Finally, it was possible to follow the Faddeev-Popov method in order to find the gauge fixed action $S_{g f}$ corresponding to the gauge fixing condition defined by the connection $\eta$.

\section{Acknowledgments}

We thank Marc Henneaux for helpful comments. 


\section{REFERENCES}

1. M. F. Atiyah and I. M. Singer, Dirac operators coupled to vector potentials, Proc. of the National Academy of Sciences of the U.S.A 81, 1984, pp. 2597-2600.

2. L. Baulieu and I. M. Singer, Topological Yang-Mills symmetry, Nucl. Phys. (Proc. Suppl.) B 5, 1988, pp. 12-19.

3. L. Baulieu and J. Thierry-Mieg, The principle of BRST symmetry: An alternative approach to Yang-Mills theories, Nucl. Phys. B 197, 1982, pp. 477-508.

4. L. Bonora and P. Cotta-Ramusino, Some Remarks on BRS Transformations, Anomalies and the Cohomology of the Lie Algebra of the Group of Gauge Transformations, Commun. Math. Phys. 87, 1983, pp. 589-603.

5. G. Catren and J. Devoto, Extended Gauge Principle in Yang-Mills Theories, to be published.

6. S. K. Donaldson and P. B. Kronheimer, The geometry of four-manifolds, Oxford University Press, 1990.

7. Dubois-Violette M., The Weil-B.R.S. algebra of a Lie algebra and the anomalous terms in gauge theory, Jour. of Geom. and Phys., Vol. 3, n. 4, 1986, pp. 525-565.

8. V. Gribov, Quantization of non-Abelian gauge theories, Nucl. Phys. B 139, 1978, pp. 1.

9. V. Guillemin, S. Sternberg, and V. W. Guillemin, Supersymmetry and equivariant de Rham theory, Spinger-Verlag, 1999.

10. M. Henneaux and C. Teitelboim, Quantization of gauge systems, Princeton Univ. Press, 1994.

11. I. Singer, Some remarks on the Gribov Ambiguity, Commun. Math. Phys. 60, 1978, pp. 7-12. 